\title{
A simulated metagenomic analysis of the gut microbiota of Anorexia Nervosa patients using PICRUSt
}

\author{
10.17975/sfj-2017-012
}

\section{AUTHORS:}

Farhaan Kanji ${ }^{\star 1}$, Himanshi Khurana ${ }^{1}$, Caitlin Sherry ${ }^{1}$ and Evangeline $\mathrm{Ng}^{1}$.

[where * indicates the primary author.]

\section{INSTITUTION:}

1. Department of Science, McMaster University, Hamilton, ON

EDITOR'S NOTE: The following was recognized as a winning project in the McMaster Indicium research competition and recommended for publication (as a graphical abstract) within the STEM Fellowship Journal. The Indicium research competition was the first undergraduate research conference hosted by one of STEM Fellowship's university branches.

\section{Abstract}

Introduction:

Mack et al. (2016) studied the fecal bacteria and archaea of 55 European normal-weight participants (NW), 55 European patients with anorexia nervosa (ANT1), and 44 ANT1 patients following a body mass index increase (ANT2). Spreadsheets of identified microbes and their relative abundance per patient were uploaded to the EBI Metagenomics web server by Mack et al. We aimed to further study the functions of the identified microbes using the PICUSt algorithm (Langille, 2013) and see if these functions are consistent with published literature.

\section{Methods:}

Spreadsheets were downloaded from EBI Metagenomics (Project\# ERP012549) in JSON Biom format and uploaded to a Galaxy cloud server hosting PICRUSt. All data transformations can be viewed at http://huttenhower.sph.harvard.edu/galaxy/u/farhaansgroup/h/anorexiastem-2017. Transformed datasets were downloaded, appended with a .biom file extension, converted to the SPF format using STAMP v2.1.3 (Parks, 2014), and merged into a single file using Microsoft Excel for analysis with STAMP. Differences in propionate metabolism between ANT1, ANT2, and NW samples was chosen for further study.

\section{Results \& Discussion:}

The proportion of propionate metabolism genes was not significantly different between ANT1 and NW samples ( $p=0.08)$, but was different between ANT2 and NW samples ( $p=0.01)$ using a pair-wise Welsh's t-test $(0.95 \mathrm{Cl})$ with a Storey FDR multiple test correction. In comparison, Mack et al, detected no differences in propionate concentration between AN and NW fecal samples using gas chromatography while Morito et al (2015) found lower concentrations of propionate in Japanese AN versus NW fecal samples using liquid chromatography.

Our discrepancy with Mack et al could have arisen since PICRUSt cannot analyze the genes of eukaryotes, PICRUSt is limited by the depth and breadth of the gene annotations in the KEGG database, and our experimental setup cannot provide data on gene expression. Moreover, $18 \%$ of V4 16S rRNA DNA sequences could not be matched to any bacteria or archaea by EBI Metagenomics. In conclusion, while in silico experiments can be useful to predict microbial functions in a sample, in this case, our PICRUSt-based hypothesis that fecal samples from Mack et al would have different concentrations of propionate between AN and NW samples was not borne out by Mack et al's chromatography experiments. Nonetheless, the conflicting findings between us, Mack et al, and Morito et al warrants further research on whether microbes mediate carbohydrate metabolism differently in patients with a history of anorexia nervosa versus controls.

\section{Keywords}

microbiome, metagenomics, anorexia nervosa, carbohydrate metabolism 


\section{A Simulated Metagenomic Analysis of the Gut Microbiota of Anorexia Nervosa Patients using PICRUSt}
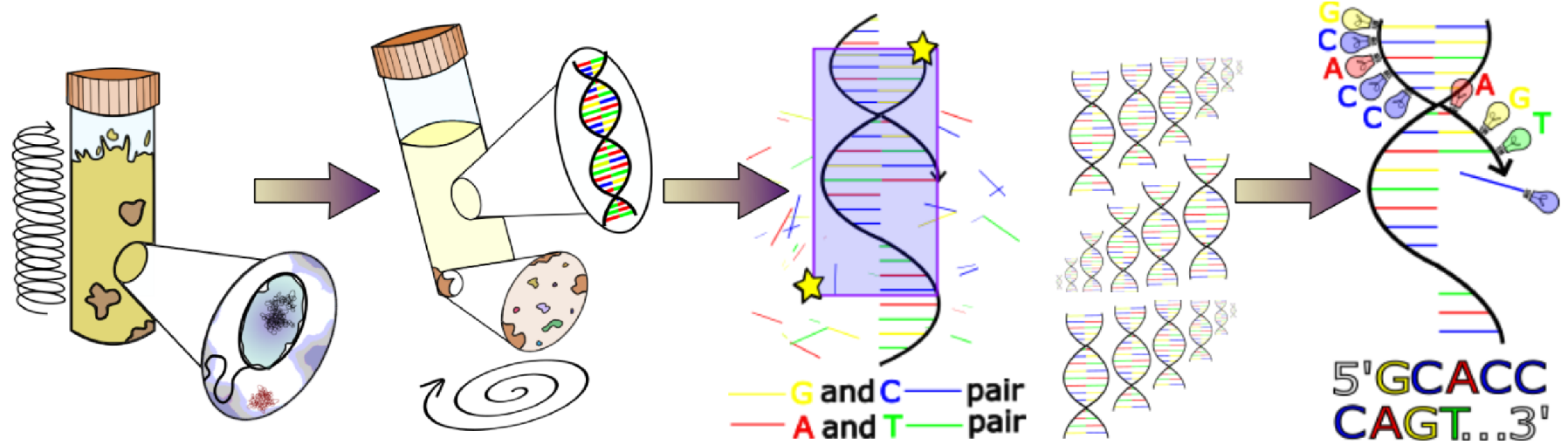

Liquification of 200 mg of fecal sample from normal-weight (NW), anorexia nervosa patients before (ANT1) or after a weight gain (ANT2)
Isolation of DNA using contaminantbinding buffers and centrifugation
Amplification of small portions of the DNA extracts corresponding to the bacterial and archaeal V4 16S rRNA gene ("V4 DNA") using PCR
The Illumina MiSeq determines the sequence of each DNA strand by copying each

DNA strand using

fluorescent dyes that can be detected by a computer

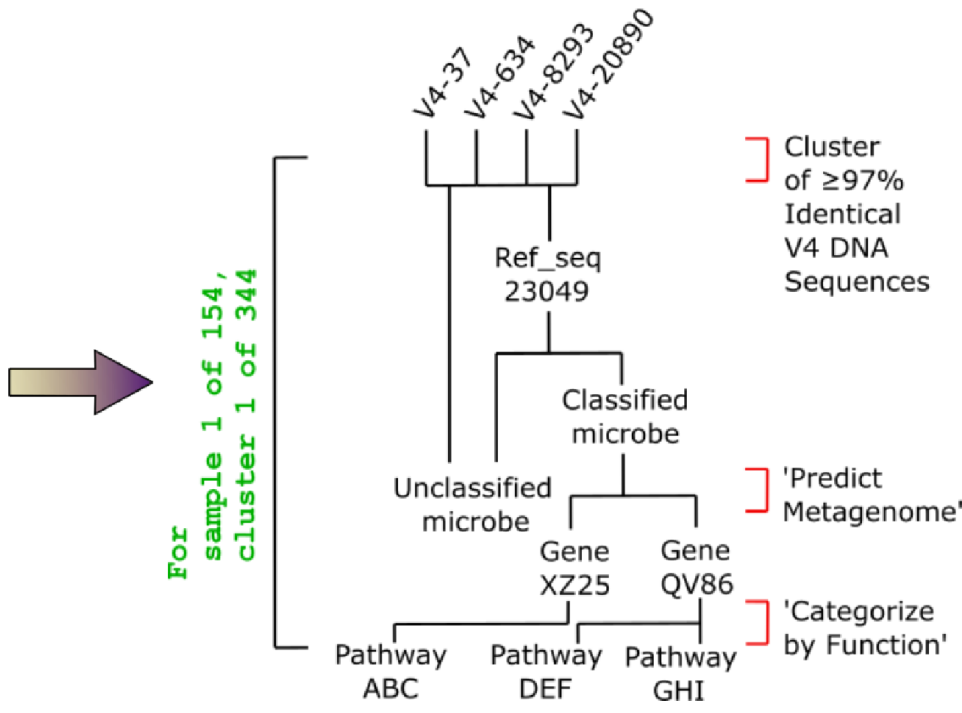

Patient-specific microbial counts were derived by matching experimentallygenerated V4 DNA sequences with classified sequences in the Greengenes database. We further analyzed the microbial counts by matching each microbe from each patient to characteristic genes catalogued in the KEGG database using PICRUSt.
Genes Involved in Propanoate Metabolism

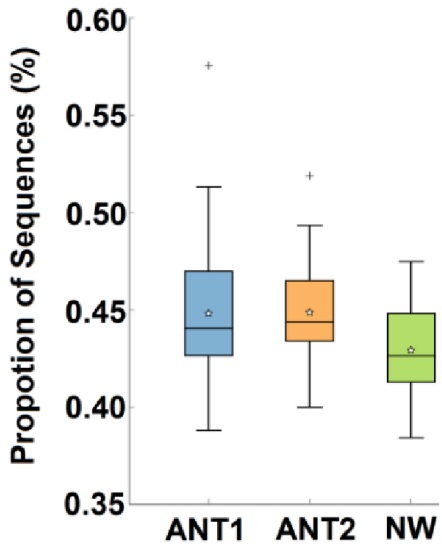

A similar proportion of identified genes in ANT1 and NW samples were involved in propanoate metabolism, but a higher proportion were found in ANT2 samples versus NW samples. In contrast, Mack et al did not measure significantly different concentrations of propanoate between fecal samples using gas chromatography. 


\section{References}

1. Mack I, Cuntz U, Grämer C, Niedermaier S, Pohl C, Schwiertz A, Zimmermann K, Zipfel S, Enck P, Penders J. Weight gain in anorexia nervosa does not ameliorate the faecal microbiota, branched chain fatty acid profiles, and gastrointestinal complaints. Scientific reports. 2016;6.

2. Langille MG, Zaneveld J, Caporaso JG, McDonald D, Knights D, Reyes JA, Clemente JC, Burkepile DE, Thurber RL, Knight R, Beiko RG. Predictive functional profiling of microbial communities using 16S rRNA marker gene sequences. Nature biotechnology. 2013 Sep 1;31(9):814-21.

3. Mitchell A, Bucchini F, Cochrane G, Denise H, Hoopen PT, Fraser M, Pesseat S, Potter S, Scheremetjew M, Sterk P, Finn RD. EBI metagenomics in 2016-an expanding and evolving resource for the analysis and archiving of metagenomic data. Nucleic acids research. 2015 Nov 17;44(D1):D595-603.

4. EBI Metagenomics [Internet]. Cambridgeshire, UK: EMBL-EBI; c2017. Microbiota composition in Anorexia Nervosa patients before and after weight gain as compared to healthy controls; 2016 October 21 [cited 2017 August 31]. Available from https://www.ebi.ac.uk/ metagenomics/projects/ERP012549

5. Afgan E, Baker D, Van den Beek M, Blankenberg D, Bouvier D, ech M, Chilton J, Clements D, Coraor N, Eberhard C, Grüning B. The Galaxy platform for accessible, reproducible and collaborative biomedical analyses: 2016 update. Nucleic acids research. 2016 May 2;44(W1):W3-10.

6. Galaxy / Hutlab [Internet]. Boston, USA: Harvard School of Public Health. Published Histories; 2017 August 21 [cited 2017 August 31];[dataset 'Anorexia_STEM-2017'] Available from http://huttenhower.sph.harvard.edu/galaxy/history/list_published

7. Parks DH, Tyson GW, Hugenholtz P, Beiko RG. STAMP: statistical analysis of taxonomic and functional profiles. Bioinformatics. 2014 Jul 23;30(21):3123-4.

8. Morita C, Tsuji H, Hata T, Gondo M, Takakura S, Kawai K, Yoshihara K, Ogata K, Nomoto K, Miyazaki K, Sudo N. Gut dysbiosis in patients with anorexia nervosa. PloS one. 2015 Dec 18;10(12):e0145274. 